\title{
Impacto del tratamiento sobre el peso y la talla en pacientes pediátricos con acidosis tubular renal en el Centenario Hospital Miguel Hidalgo
}

Cendejas-Ayala María de Lourdes *, Orozco-Loza Iraida Luz *, Barajas-Salcedo Gerardo*, Rosas-Cabral Alejandro **

\begin{tabular}{|c|c|}
\hline 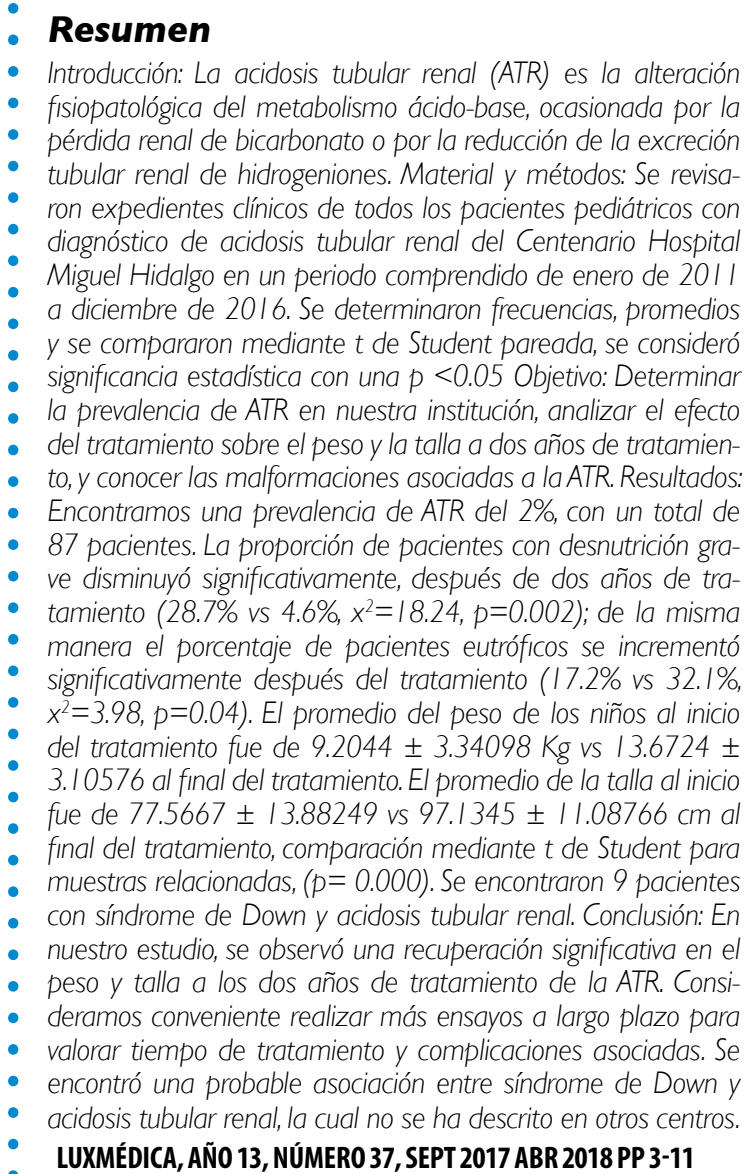 & 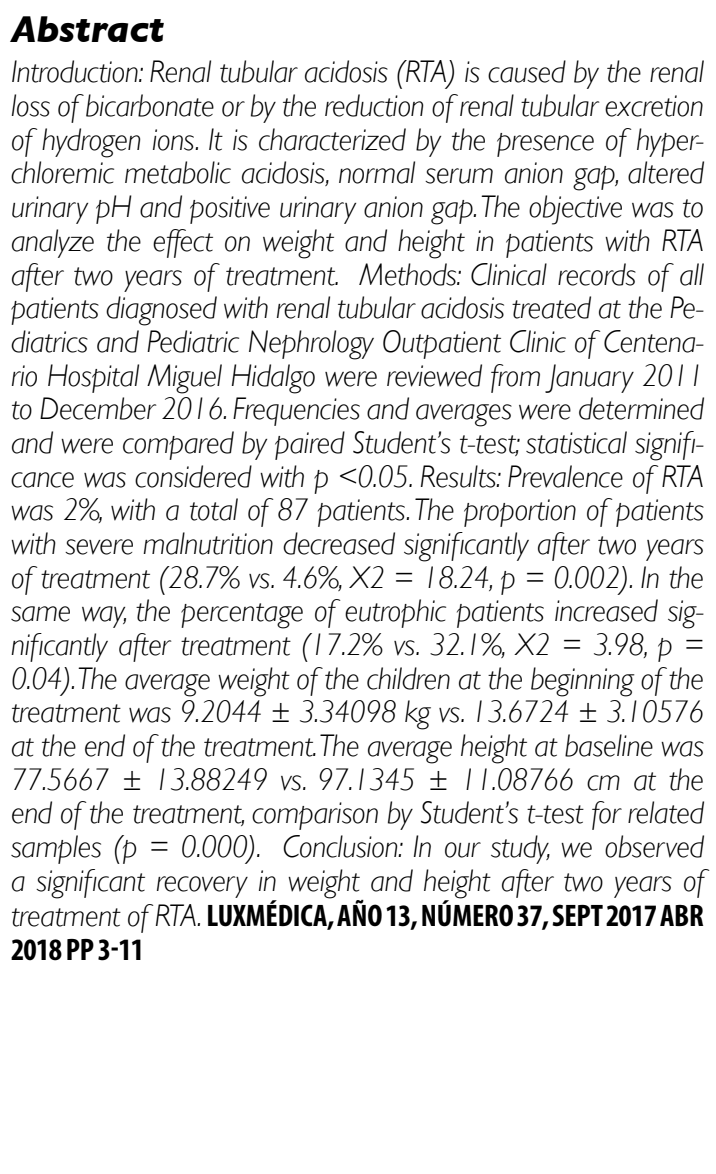 \\
\hline Palabr & \\
\hline
\end{tabular}

* Médicos adscritos al Departamento de Pediatría del Centenario Hospital Miguel Hidalgo

** Profesor Investigador del Departamento de Medicina del Centro de Ciencias de la Salud de la Universidad Autónoma de Aguascalientes

Fecha de recibido: 10 de julio 2017

Fecha de aceptación: 15 enero 2018

Correspondencia: Alejandro Rosas Cabral. Departamento de Medicina. Edificio 107, planta alta. Campus Univesitario. Universidad Autónoma de Aguascalientes. Av. Universidad 940, Ciudad Universitaria. Código postal 20131. Teléfono +52 (449) 9108443 Aguascalientes, Aguascalientes, México. Correo electrónico drrosascabral@hotmail.com 


\section{Introducción}

La acidosis tubular renal (ATR) es una alteración fisiopatológica del metabolismo ácido-base caracterizada por la presencia de acidosis metabólica hiperclorémica, debida a la pérdida renal de bicarbonato o por la reducción de la excreción tubular renal de hidrogeniones. ${ }^{1,2}$

La acidosis tubular renal (ATR) se clasifica en ATR proximal o tipo 2 cuando existe una alteración en la reabsorción del bicarbonato en el túbulo proximal de la nefrona. En tipo 1 o distal, si se encuentra disminución en la secreción neta de hidrogeniones en el túbulo colector renal, lo cual produce un $\mathrm{pH}$ urinario $>5.5$; y la ATR hiperkalémica (o tipo 4). Anteriormente se utilizaba la categoría ATR tipo 3, en niños con ATR distal tipo 1, quienes en la edad de la lactancia presentan también pérdida proximal de bicarbonato en orina de manera transitoria, sin embargo se retiró de la clasificación.

Normalmente alrededor del 75 al $80 \%$ del bicarbonato filtrado es reabsorbido, por consiguiente "devuelto" a la sangre por el túbulo proximal de la nefrona. Si la capacidad de reabsorción del túbulo renal se reduce (como en ATR proximal primaria y síndrome de Fanconi), ocurrirá liberación aumentada del bicarbonato en segmentos distales de la misma, lo cual sobrepasa la capacidad de absorción del bicarbonato, produciendo bicarbonaturia y acidosis metabólica. ${ }^{3}$ El tratamiento de los pacientes con esta variedad de ATR debe ser con suplemento de bicarbonato. En los niños, en particular, la terapia con reemplazo de bicarbonato es crítico para la prevención del retraso del crecimiento debido a la acidosis, requiriendo dosis elevadas de bicarbonato para lograr su control (5-15 $\mathrm{mEq} / \mathrm{kg}$ de peso corporal); además de la adición de un diurético tiazídico, ya que mejora la reabsorción en el túbulo proximal de bicarbonato. 4,5 La combinación anterior puede agravar la hipokalemia, debido a la secreción de $\mathrm{K}+$ en el túbulo colector cortical (CCT) como resultado de una mayor entrega distal de bicarbonato.

La acidosis tubular renal distal (ATRd) es una enfermedad renal rara y compleja debido a un defecto en la excreción de la carga ácida $(\mathrm{H}+$ y NH4) en las células alfa del conducto colector. La acumulación de la carga ácida es resultado de la tasa entre el consumo y la reducción del bicarbonato en la nefrona distal y el CO2 que funciona como amortiguador en la sangre..$^{6-10}$ Los rasgos clínicos característicos de ATRd son la presencia de acidosis sistémica junto con la incapacidad para acidificar la orina a un $\mathrm{pH}<5.3$, asociada con defectos en la reabsorción de $\mathrm{K}+\mathrm{y}$ a hipercalciuria (ya que la acidosis crónica disminuye la reabsorción de $\mathrm{Ca}++) .{ }^{7}$ La enfermedad debuta en los primeros años de vida. ${ }^{8}$ Se caracteriza por presencia de vómito, poliuria, deshidratación y debilidad muscular debido a la hipokalemia, la cual se produce por movilización de éste hacia el espacio intracelular, secundario a la presencia de acidosis 
metabólica y a la pérdida urinaria de potasio a fin de mantener la electroneutralidad sobre la membrana apical. Litiasis urinarias y nefrocalcinosis por la hipercalciuria, hipocitraturia y la presencia de orina alcalina. Retraso del crecimiento por raquitismo (también secundario a la hipercalciuria). ${ }^{6,8}$ El objetivo principal de cualquier tratamiento para ATRd es revertir la acidosis, lo que reduce la calciuria y aumenta simultáneamente la excreción de citrato. Esto conduce a un menor riesgo de nefrolitiasis y osteoporosis. Actualmente el citrato de potasio a dosis de 1-2 mEq / kg / día, es el tratamiento de elección. Con el citrato de potasio, se proporciona no sólo un donante de bicarbonato para tratar la acidosis, sino que también se compensa la pérdida de potasio de manera simultánea, además parece tener efectos positivos sobre la densidad mineral ósea y la función celular ósea. ${ }^{7}$ Es importante la cuantificación de calcio en orina de 24 horas (valor normal $<4 \mathrm{mg} / \mathrm{kg} /$ día) para la prevención de nefrocalcinosis. ${ }^{3}$

La alergia a las proteínas de la leche de vaca es una alteración con alta prevalencia mundial, se estima que entre 1 y $12 \%$ de la población la padece. La alergia a las proteínas de la leche de vaca es la causa más común de alergia a alimentos en lactantes. Se define como una reacción inmunitaria a las proteínas de la leche de vaca, acompañada de signos y síntomas clínicos. Las reacciones adversas a los alimentos pueden ser de dos tipos: la primera es la hipersensibilidad con respuesta inmunitaria mediada por IgE y representa de 10 a $25 \%$ de los casos. El segundo tipo de reacciones son las no mediadas por IgE, que son las más frecuentes y representan de 75 a $90 \%$ de los casos. En el Instituto Nacional de Pediatría se realizó un estudio entre marzo de 2008 y noviembre de 2009, en donde se encontró que el $33 \%$ de los pacientes con alergia a la proteína de la leche de vaca presentaban además acidosis tubular renal. ${ }^{11}$

En los últimos años se han publicado diversos estudios en los que se demuestra la relación que existe entre la desnutrición grave y ciertas alteraciones funcionales y estructurales. ${ }^{12}$ La acidosis tubular renal es un problema de salud a nivel internacional debido a que condiciona detención del crecimiento y falla en el medro. A nivel internacional se reporta una incidencia de 1 caso por cada 1 millón de habitantes. Topaloglu y cols. Garcia y cols reportan una prevalencia de 35 casos por cada 10,000 expedientes revisados en un estudio realizado en el Instituto Nacional de Pediatría. ${ }^{2}$ En el estado de Aguascalientes no existen datos reportados sobre la prevalencia de esta patología.

Se considera que con el tratamiento de la acidosis se remite la sintomatología lo cual condicionará una mejoría en el crecimiento, tanto en ganancia ponderal como mejoría en la talla. No se cuenta con estudios que valoren la respuesta al tratamiento sobre la relación peso/talla como marcador de desnutrición en nuestro estado, por lo que nosotros nos propusimos evaluar el peso y la talla a 
dos años del tratamiento en los niños con diagnóstico de acidosis tubular renal proximal y distal en el Centenario Hospital Miguel Hidalgo. El objetivo de este trabajo fue determinar la prevalencia de la acidosis tubular renal, y establecer cuáles son las malformaciones asociadas a acidosis tubular renal en la población pediátrica del Centenario Hospital Miguel Hidalgo.

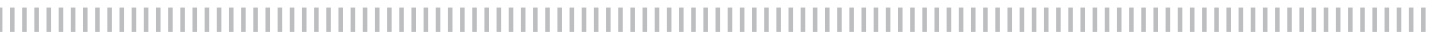

\section{Material y métodos}

Se realizó un estudio transversal mediante la revisión de los expedientes de un total de 4,350 pacientes atendidos en la consulta externa de Pediatría y Nefrología Pediátrica del Centenario Hospital Miguel Hidalgo entre enero de 2011 y diciembre de 2016, para determinar el número de pacientes con acidosis tubular renal proximal o distal. Así mismo, realizamos un estudio longitudinal para determinar el efecto del tratamiento con soluciones de bicarbonato o citratos durante dos años, sobre el peso y la talla de los pacientes con diagnóstico de ATR de nuestro hospital.

A los pacientes seleccionados se les administró tratamiento con: a) Solución de bicarbonato: bicarbonato de sodio $43 \mathrm{gr}$, bicarbonato de potasio $53 \mathrm{~g}$, fosforo y vitamina $D$ normales, agua $500 \mathrm{ml}(1 \mathrm{mEq}$ de sodio, $1 \mathrm{mEq}$ de potasio y $2 \mathrm{mEq}$ de bicarbonato por $\mathrm{ml}$ ) b) Solución de citratos: ácido cítrico 70 gr, citrato de sodio 98 gr, citrato de potasio $108 \mathrm{gr}$, agua y jarabe de grosella 1,000 ml ( $1 \mathrm{mEq}$ de sodio, $1 \mathrm{mEq}$ de potasio y $2 \mathrm{mEq}$ de citrato por $\mathrm{ml}$ ), durante dos años seguidos. ${ }^{3}$

La administración de cada uno de los diferentes tratamientos no fue de acuerdo al diagnóstico de ATR distal o proximal, sino a la tolerancia del mismo por los pacientes.

Las variables estudiadas fueron peso y talla antes y después del inicio del tratamiento, malformaciones congénitas asociadas y presencia de alergia a las proteínas de la leche vacuna. Se estableció el diagnóstico de desnutrición según las tablas de relación peso/talla de niños de 0 a 6 años de la OMS y de IMC para niños de 6 a 19 años de la OMS, no se emplearon tablas específicas para niños con Síndrome de Down.

El análisis estadístico se realizó determinando frecuencia, media, desviación estándar, prueba de Kolmogorov-Smirnov para bondad de ajuste y demostrar normalidad de las variables a estudiar, y prueba de $t$ de Student para muestras relacionadas. Se consideró significancia estadística con una $p<0.05$, con dos colas.

\section{| | | | | | | | | | | | | | | | | | | | | | | | | | | | | | | | | | | | | | | | | | | | | | | | | | | | | | | | | | | | | | | | | | | | | | | | | | | | | | | | | | | | | | | | | | | | | | | | | | | | | | | | | | | | | | | | | ||}

\section{Resultados}

De 4,350 pacientes atendidos en la consulta externa de Pediatría y Nefrología Pediátrica del Centenario Hospital Miguel Hidalgo entre 2011 y 2016; se encontraron 107 pacientes con sospecha diagnóstica de Acidosis Tubular Renal, de los cuales se excluyeron 20 por no cumplir con los criterios de inclusión, para un total de 87 pacientes incluidos en el estudio, lo cual representa una prevalencia del $2 \%$ en nuestra población.

En cuanto a la distribución por sexos, la ATR se observó más frecuentemente en el sexo femenino con 46 casos, representando el $52.9 \%$ (tabla 1$)$. En el $73.6 \%(n=64)$ de nuestros pacientes se realizó el diagnóstico de acidosis tubular renal y se inició tratamiento médico dentro de los primeros 2 años de vida. 
Se calculó en todos los pacientes la tasa de filtración glomerular por medio de la fórmula de Schwartz, de los cuales el $64.4 \%(n=56)$ se encontraron dentro de los límites esperados para la edad (tabla 1).

53 pacientes no tuvieron malformaciones asociadas, en 9 pacientes se encontró Síndrome de Down, 5 con cardiopatías tratadas con manejo anticongestivo, y en 20 de ellos se encontraron otras malformaciones como estenosis uretero-piélica, monorreno, o reflujo vesico-ureteral (tabla 1).

En cuanto a la asociación de alergia a la proteína de la leche de vaca y acidosis tubular renal, encontramos que únicamente 4 de nuestros pacientes presentaron esta asociación, representando el 5\% (tabla 1).

\section{Tabla I}

\section{Características demográficas en 87 pacientes con ATR del Centenario Hospital Miguel Hidalgo}

\begin{tabular}{|c|c|c|}
\hline Sexo & $\mathbf{N}$ & $\%$ \\
\hline Mujeres & 46 & 52.9 \\
\hline Hombres & 41 & 47.1 \\
\hline \multicolumn{3}{|c|}{ Tasa de filtración glomerular } \\
\hline Normal & 56 & 64.4 \\
\hline Anormal & 31 & 35.6 \\
\hline \multicolumn{3}{|c|}{ Alergia a proteína de la leche de vaca } \\
\hline Sí & 4 & 5 \\
\hline No & 83 & 95 \\
\hline \multicolumn{3}{|l|}{ Tratamiento otorgado } \\
\hline Solución de bicarbonato & 33 & 38.0 \\
\hline Solución de citratos & 25 & 28.7 \\
\hline Ambas soluciones & 29 & 33.3 \\
\hline \multicolumn{3}{|l|}{ Malformación } \\
\hline Ninguna & 53 & 61 \\
\hline Síndrome de Down & 9 & 10 \\
\hline Cardiopatías & 5 & 6 \\
\hline Otras & 20 & 23 \\
\hline
\end{tabular}

En relación al tipo de tratamiento administrado en los pacientes $38 \% \quad(n=33)$ fueron tratados con bicarbonato, $28.7 \%$ $(n=25)$ con citratos y el $33.3 \% \quad(n=29)$ con ambos al mismo tiempo.

Se analizó el estado de nutrición de los pacientes al diagnóstico e inicio del tratamiento, en el cual la mayoría de estos se encontraron en algún grado de desnutrición, con un $78.1 \%(n=68)$. La distribu- ción de los pacientes según su estado nutricional fue: 25 con desnutrición grave, 32 con desnutrición moderada, 11 con desnutrición leve, 4 con eutrofia, 2 con sobrepeso y 2 con obesidad, al inicio del tratamiento. La distribución a dos años de tratamiento fue: 4 con desnutrición grave, 33 con desnutrición moderada, 21 con desnutrición leve, 28 eutróficos y uno con sobrepeso (tabla 2 ). 


\section{Tabla 2}

\section{Comparación del estado nutricional antes y después de dos años de tratamiento en 87 niños con ATR.}

\begin{tabular}{|lccc|}
\hline Estado nutricional & Antes del tratamiento & Después del tratamiento & $\mathrm{P}$ \\
\hline Desnutrición grave & 25 & 4 & 0.002 \\
\hline Desnutrición moderada & 32 & 33 & $\mathrm{NS}$ \\
\hline Desnutrición leve & 11 & 21 & $\mathrm{NS}$ \\
\hline Eutróficos & 15 & 28 & 0.04 \\
\hline Sobrepeso & 2 & 1 & $\mathrm{NS}$ \\
\hline Obesidad & 2 & 0 & $\mathrm{NS}$ \\
\hline
\end{tabular}

La proporción de pacientes con desnutrición grave disminuyó significativamente, después de dos años de tratamiento ( $28.7 \%$ vs $\left.4.6 \%, x^{2}=18.24, p=0.002\right)$; de la misma manera el porcentaje de pacientes eutróficos se incrementó significativamente después del tratamiento $(17.2 \%$ vs $\left.32.1 \%, x^{2}=3.98, p=0.04\right)$. El promedio del peso de los niños al inicio del tratamiento fue de $9.2044 \pm 3.34098 \mathrm{Kg}$ vs 13.6724 \pm 3.10576 al final del tratamiento. El promedio de la talla al inicio fue de 77.5667 \pm 13.88249 vs $97.1345 \pm 11.08766 \mathrm{~cm}$ al final del tratamiento, comparación mediante $t$ de Student para muestras relacionadas, $(p=0.001)$

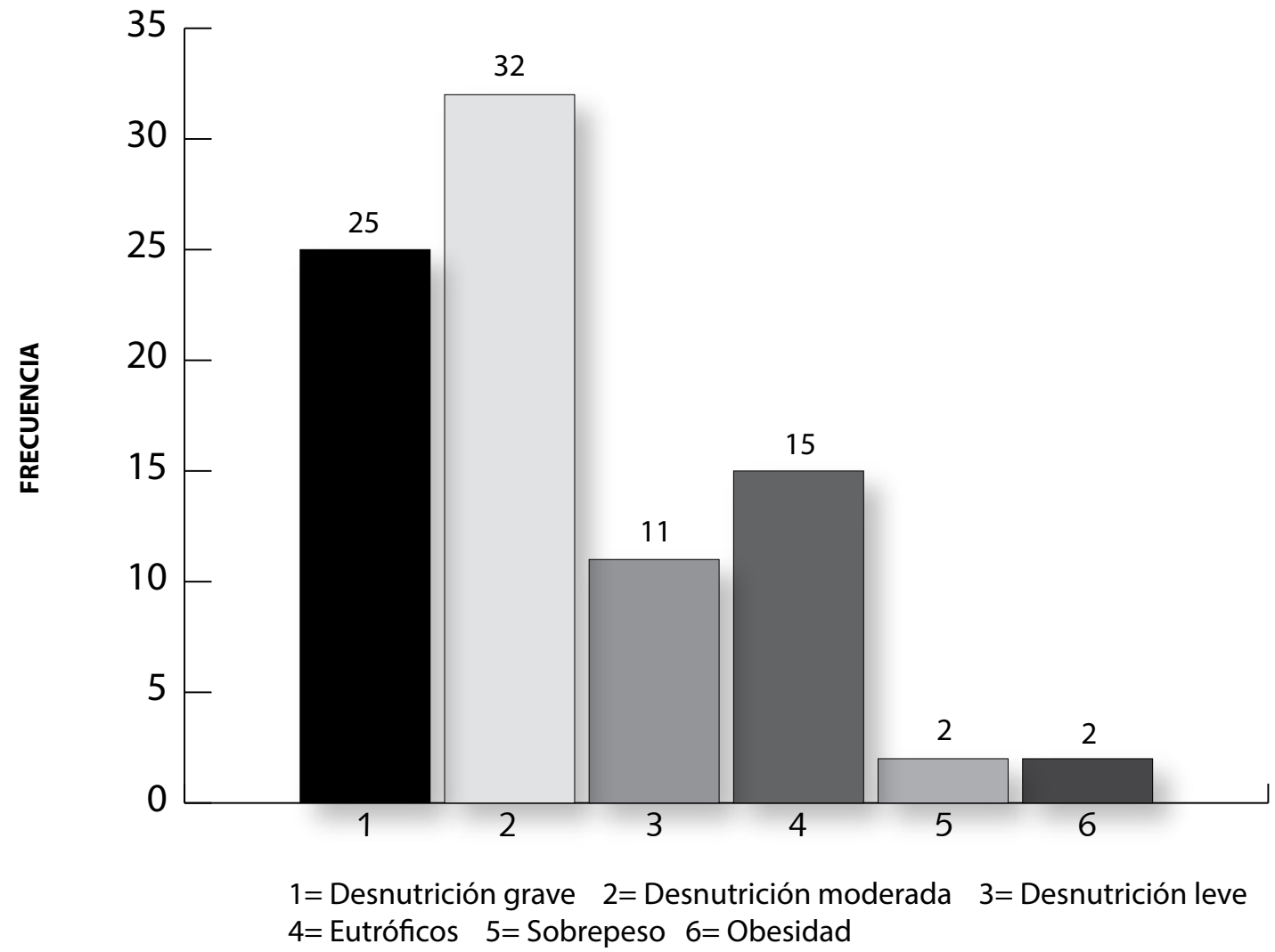

Figura 1. Estado nutricional de 87 pacientes con ATR del Centenario Hospital Miguel Hidalgo previo a tratamiento con soluciones de bicarbonato y/o citrato. 


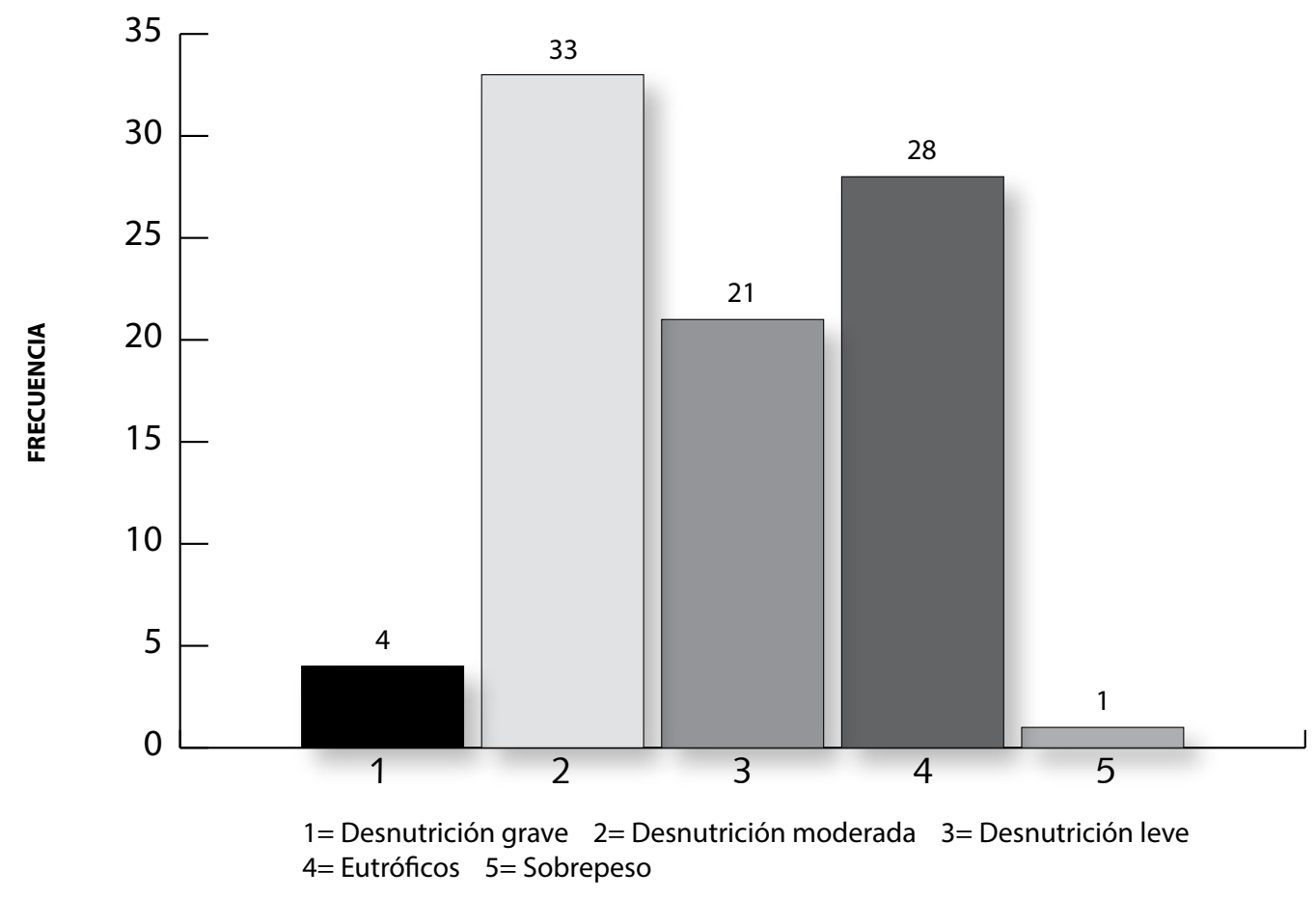

Figura 2. Estado nutricional de 87 pacientes con ATR del Centenario Hospital Miguel Hidalgo posterior a tratamiento con soluciones de bicarbonato y/o citrato.

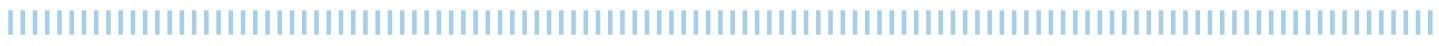

\section{Discusión}

La acidosis tubular renal representa un problema frecuente en los pacientes hospitalizados tanto adultos como pediátricos a nivel mundial, ya sea como complicación de alguna otra patología o bien asociada a alteraciones hereditarias. ${ }^{13}$ Los trastornos hereditarios que afectan a los túbulos renales, aunque constituyen un grupo de enfermedades raras, resultan en alteraciones en la homeostasis acido-base. ${ }^{14}$ La sintomatología asociada a la acidosis tubular renal es altamente inespecífica, no obstante constituye un grupo de enfermedades a considerar siempre en los niños con alteraciones en el medro ${ }^{15}$ y cuya frecuencia es controversial debido a que tanto a ni- vel internacional como nacional se refieren frecuencias elevadas de la misma, lo cual puede ser debido a los diferentes métodos de laboratorio empleados por los diversos grupos de investigación. ${ }^{16}$ Así, por ejemplo, en nuestro país algunos autores han referido que se realiza un diagnóstico incrementado de estos padecimientos y que pudieran estar relacionados con el tipo de pruebas de laboratorio en las que se basa el diagnóstico. ${ }^{17}$

Nosotros encontramos una prevalencia del $2 \%$ en nuestra población, lo cual resulta claramente superior a lo referido por Guerra y colaboradores, quienes solo encontraron cinco pacientes con esta patología. ${ }^{18}$ Sin embargo, la consideramos también una 
causa importante de desnutrición en nuestros pacientes. Es de vital importancia tener esta patología presente en el abordaje de los niños con desnutrición, ya que varios de estos pacientes se encuentran subdiagnosticados, debido a su tan inespecífica sintomatología: náuseas, vómito, hiporexia y falla en el medro, siendo tal vez esta una de las razones por la que la incidencia a nivel mundial y nacional es tan disímbola. Los principales criterios paraclínicos para el diagnóstico de ATR son: acidosis metabólica hiperclorémica, alteraciones en el $\mathrm{pH}$ urinario, anion gap sérico normal y anion gap urinario positivo; en estos pacientes es importante conocer la fracción excretada de bicarbonato y la realización de la prueba de carga de cloruro de amonio para integrar un diagnóstico más certero de esta patología, sin embargo en nuestro medio no se encuentran disponibles en la mayor parte de los hospitales, y únicamente algunos centros de atención terciaria cuentan con ellos. La mayor proporción de los pacientes al momento del diagnóstico se concentraron entre los 12 y 24 meses de edad, lo cual concuerda con lo referido en la literatura de ser más frecuente durante el primer año de vida. En nuestros pacientes es conveniente hacer el diagnóstico de manera más oportuna para evitar que presenten un estado de desnutrición tan avanzado, así como para prevenir complicaciones, siendo la nefrocalcinosis la más importante de ellas. En la literatura se refiere que la ATR es en la mayoría de los casos autolimitada, sin embargo, no se hace referencia del tiempo ideal para la duración del tratamiento; aunque este no fue el objetivo de nuestro estudio, encontramos pacientes que continuaron recibiendo tratamiento aún cinco años después de su diagnóstico, por persistir con acidosis metabólica hiperclorémica. Consideramos importante la búsqueda intencionada de alergia a la proteína de la leche de la vaca, ya que se encuentra descrito un gran porcentaje de asociación de ATR y enfermedades alérgicas, ${ }^{19}$ sin embargo en nuestra población, todos los hallazgos fueron incidentales, a diferencia de los grandes institutos donde estos pacientes son valorados por el servicio de gastroenterología para descartar esta patología con múltiples paraclínicos. Se comenta que los pacientes con acidosis tubular renal presentan recuperación en cuanto al peso y talla al iniciar el tratamiento médico, sin embargo no se hace referencia al tiempo de duración del tratamiento y recuperación del estado eutrófico, en nuestro estudio sí se observó una recuperación en el peso y talla a los dos años de revaloración, sin embargo aún no se había recuperado al $100 \%$ el estado eutrófico de los pacientes, por lo que sería conveniente continuar su vigilancia a largo plazo.

\section{Conclusiones}

Se muestra una prevalencia del $2 \%$ en nuestra población de estudio. Al inicio del estudio del $78.1 \%(n=87)$ se encontraban en algún grado de desnutrición, únicamente $14.8 \%(n=25)$ de pacientes con desnutrición grave, permanecen en este estado a los dos años del tratamiento. Se observó una recuperación estadísticamente significativa en cuanto al peso y talla de nuestros pacientes a los dos años de iniciado el tratamiento. La malformación congénita aislada más frecuentemente observada en nuestros pacientes fue el Síndrome de Down. 


\section{Bibliografía}

1. Santos F, Gil-Peña H, Álvarez-Álvarez S. Renal tubular acidosis. Curr Opin Pediatr. 2017; 29(2):206210.

2. Muñoz AR, Escobar L, Medeiros M. Acidosis tubular renal en niños: conceptos actuales de diagnóstico y tratamiento. Bol Med Hosp Infant Mex . 2013; 70(3):178-194.

3. Velázquez Jones. Acidosis tubular renal. Bol Med Hosp Infant Mex 2012; 69(6):502-508.

4. Ring T, Frische S, Nielsen S. Clinical review: Renal tubular acidosis - a physicochemical approach. Critical Care. 2005; 9:573-580.

5. Haque S, Ariceta G, Batlle D. Proximal renal tubular acidosis: a not so rare disorder of multiple etiologies. Nephrol Dial Transplant. 2012; 27: 4273-4287

6. Barros $P C$, Medeiros $M F$, Cunha $M L$, Araújo $O E$, Marques MD, Simões SA. Whole-exome sequencing as a diagnostic tool for distal renal tubular acidosis. J Pediatr. 2015; 91(6): 583-9.

7. Both $T$, Zietse $R$, Hoorn EJ, Martin VH, Virgil AS, Dalm JA et al. Everything you need to know about distal renal tubular acidosis in autoimmune disease. Rheumatol Int. 2014; 34:1037-1045

8. Guías clínicas de padecimientos más frecuentes en Nefrología Pediátrica. Departamento de Nefrología. Hospital Infantil Federico Gómez.

9. Honore PM, Jacobs R, Hendrickx I, De Waele E, Gorp V, Spapen HD. Renal tubular acidosis with hyperchloremic acidosis: harmless with a sting? Critical Care . 2015; 19:305.

10. Escobar L, Mejía N, Gil H, Santos F. La acidosis tubular renal distal: una enfermedad hereditaria en la que no se pueden eliminar los hidrogeniones. Nefrología 2013; 33(3):289-96.

11. Cervantes RB, Zapata CC, Hernández VB, Zárate MF, Sánchez PM, Montijo BE et al. Utilidad de las diferentes pruebas diagnósticas para alergia a las proteínas de la leche de vaca y su asociación con aci- dosis tubular renal. Rev Enfer Infec Pediatr. 2011; 24(96):147-153

12. Anjum M, Moorani KN, Sameen I, Ayaz MM, Kulsoom S. Functional and structural abnormalities of the kidney and urinary tract in severely malnourished children - A hospital based study. Pak J Med Sci. 2016; 32(5): 1135- 1140.

13. Brunner R, Drolz A, Scherzer TM, Staufer K, Fuhrmann V, Zauner C, Holzinger U, Schneeweil B. Renal tubular acidosis is highly prevalent in critically ill patients. Crit Care. 2015;19:148.

14. Palazzo V, Provenzano A, Becherucci $F$, Sansavini $G$, Mazzinghi $B$, Orlandini $\vee$ et al. The genetic and clinical spectrum of a large cohort of patients with distal acidosis tubular renal. Kidney Int. 2017; 91(5): 1243-1255.

15. Adedoyin O, Gottlieb B, Frank R, Vento S, Vergara $M$, gauthier $B$, Trachtman $H$. Evaluation of failure to thrive: diagnostic yield of testing for renal tubular acidosis. Pediatrics. 2003; 112(6) Pt 1): e463.

16. Topaglu R, Baskim E, Bahat E, Kavukcu S, Cakar N, Donmez O, Gur Guven A, Caliskan S, Erdogan O, Yalcinkaya $F$. Hereditary renal tubular acidosis in Turkey: demographic, clinical, and laboratory features. Clin Exp Nephrol. 2011;15:108-111.

17. Muñoz-Arizpe R, Escobar L, Medeiros M. Over-diagnosis of renal tubular acidosis in Mexico. Rev Invest Clin. 2012;64(4):399-401.

18. Guerra-Hernández $N$, Matos-Martinez $M$, OrdazLopez KV, Camargo-Muñiz MD, Medeiros DM, Escobar-Perez L. Clinical and biochemical findings in Mexican patients with distal renal tubular acidosis. Rev Invest Clin. 2014;66(5):386-392.

19. Morfín-Maciel BM, García De la Puente S, Bojórquez-Ochoa A, Huante-Anaya A, Orozco-Martínez $\mathrm{S}$, Zaltzman-Girshevich S, Guzmán-Vázquez E. Prevalence of allergic diseases in children with secondary renal tubular acidosis attending a tertiary care pediatric center. Rev Alerg Mex. 2017 64(2): 133141. 